TRABAJOS DE PREHISTORIA

$51,{ }^{\circ}{ }^{\circ} 1,1994$, pp. $111-125$

\section{APROXIMACIÓN AL ESTUDIO ETNOARQUEOLÓGICO DEL GUADIANA MENOR (JAÉN)}

\author{
APPROACHES \\ TO ETHNOARCHAEOLOGY IN THE \\ GUADIANA MENOR VALLEY (JAÉN)
}

\author{
MACARENA FERNÁNDEZ RODRÍGUEZ $(*)$ \\ FRANCISCO JAVIER LÓPEZ FERNÁNDEZ (*) \\ ANTONIO MADRIGAL BELINCHÓN (**) \\ VICTORINO MAYORAL HERRERA $(* *)$
}

\section{RESUMEN}

La Etnoarqueología no ha sido habitualmente utilizada en España, probablemente por falta de reconocimiento de un nexo con un pasado remoto. Sin embargo, consideramos que en ciertas áreas apartadas y con recursos limitados, han podido mantenerse formas de vida básicamente similares, al menos desde fines de la Edad del Hierro hasta hace relativamente poco tiempo. Los testimonios ofrecidos por las personas que vivieron en esas condiciones son de gran ayuda para comprender y comparar la organización y el uso del paisaje a través del tiempo. En este trabajo se analiza un área centrada en el Valle del Guadiana Menor, a partir de fuentes arqueológicas, medievales y recientes.

\begin{abstract}
Ethnoarchaeology has not been employed very often in Spain, probably because we do not recognise any link with our remote past. However, we think that some marginal areas with limited resources have maintained a quite similar way of life at least from the Iron Age until the re-

(*) Museos municipales de Ciudad Real.

(**) Departamento de Prehistoria. Facultad de Geografía e Historia. Universidad Complutense. 28040 Madrid.

El artículo fue remitido en su versión final el 17-I-94.
\end{abstract}

cent past. Oral evidence given by the people that lived under those conditions is of great help in order to understand and compare the organisation and use of the landscape through time. In this paper we begin the study of an area centered on the Guadiana Menor River Valley, using archaeological data (Iron Age), and medieval and recent written sources.

Palabras clave: Etnoarqueología. Cultura ibérica. Hábitat Rural. Economía. Guadiana Menor.

Key words: Ethnoarchaeology. Iberian culture. Rural Settlement. Economy. Guadiana Menor.

\section{INTRODUCCIÓN}

$\mathrm{Al}$ igual que ocurre en muchos otros países del ámbito mediterráneo (por referirnos sólo a nuestro entorno más inmediato), y debido en gran medida a una incorporación a un proceso de industrialización muy desigual y en general retardado, en España existen aún amplias zonas en las que las formas económicas y sociales del mundo rural tradicional se han mantenido hasta 
tiempos muy recientes. Sólo a partir de los últimos treinta años estos lugares han empezado a romper su aislamiento, y han sufrido una fuerte transformación que ha implicado una radical modificación de los modos de vida de sus habitantes.

El núcleo fundamental de dichas formas de vida ha sido una economía campesina sumamente autárquica, unos recursos tecnológicos de carácter pre-industrial y una estrecha adaptación a un entorno ambiental que generalmente presenta fuertes limitaciones.

El valle del Guadiana Menor, zona en la que vamos a centrar nuestro estudio, ofrece todas estas características. Desde 1983 se vienen realizando allí investigaciones arqueológicas en el contexto de un proyecto denominado "El poblamiento ibérico en el valle del Guadiana Menor", dirigido por los doctores Teresa Chapa Brunet y Juan Pereira Sieso, cuyo centro principal de intervención es la necrópolis y el poblado ibéricos de Los Castellones de Ceal (Hinojares, Jaén).

Diez años de excavaciones han facilitado un intenso contacto con las gentes del lugar. A través de su testimonio y opiniones fue como empezamos a percibir la gran riqueza etnográfica que aún conservaba la región, y la participación de algunas de estas personas en los trabajos de campo nos sensibilizó además sobre el valor potencial de este patrimonio para la contrastación de problemas surgidos en el curso de la excavación arqueológica. Las crecientes evidencias de una escasa modificación del medio natural en los últimos dos milenios fueron un incentivo más para plantear la posibilidad de que procesos adaptativos desarrollados en época ibérica pudieran ser mejor comprendidos a la luz del conocimiento de las adaptaciones propias de la vida rural tradicional que ahora se extingue.

A la hora de emprender nuestro estudio, y como esperamos argumentar suficientemente en el curso de este artículo, consideramos la aproximación metodológica de la Etnoarqueología como el camino más productivo y estimulante. Se impone, antes de continuar, una breve reflexión sobre este concepto, que será al tiempo un intento de clarificación sobre nuestras expectativas y objetivos.

\section{EN TORNO AL CONCEPTO DE ETNOARQUEOLOGIA}

Al compás de los sucesivos y acentuados movimientos pendulares que ha experimentado la teoría arqueológica desde sus inicios, la información etnográfica ha sido objeto de un tratamiento muy desigual. Por encima de todo hay que resaltar la gran frecuencia con que se ha empleado para establecer analogías sin una seria consideración de los contextos ambientales, culturales e históricos de los datos que se pretendían extrapolar hacia el pasado.

Durante toda la década de los sesenta y setenta los planteamientos de la arqueología tradicional fueron sometidos a una intensa labor de crítica. En el marco de la grave crisis que esta corriente provoca nace la necesidad de desarrollar nuevos puntos de referencia teórica, y la Etnoarqueología, que tiene como verdadero punto de arranque este momento, juega un importante papel al respecto al menos en dos sentidos principales.

En primer lugar, debido al notable cambio operado en los objetivos de la Arqueología, se produjo un gran vacío teórico que indujo a muchos a tomar prestados modelos e ideas directamente de la teoría antropológica (del mismo modo que se hizo con otras disciplinas como la Geografía o las Ciencias Naturales). De este modo se crearon relaciones de dependencia (Gould, 1980: 2) en perjuicio de la vitalidad y validez de un enfoque específicamente arqueológico. Ulteriores desarrollos por este camino han llevado a algunos arqueólogos a tomar parte activa en la utilización de datos y procedimientos etnográficos, fomentando de este modo la creación de verdaderos estudios interdisciplinares.

Por otra parte, y en íntima relación con las mencionadas transformaciones, fueron planteados nuevos desafíos metodológicos, al cuestionarse la posibilidad de que la Arqueología lograra extraer del registro material pautas que aportaran información significativa sobre cualquier aspecto del comportamiento humano.

Así, autores como Schiffer (1976) o Binford (1977, 1981) (recogidos en Trigger, 1992: 336) han intentado tratar de un modo sistemático la especificidad de los datos arqueológicos, así como la creación de técnicas concretas para comprender las relaciones entre estos y los complejos sistemas humanos en funciona- 
miento. Esto es lo que da sentido a la propuesta binfordiana del establecimiento de una Teoría de Alcance Medio, esto es, de sistemas para investigar de que modo factores adaptativos, culturales o no intencionales influyen en las distribuciones de artefactos documentadas por los arqueólogos. Respecto a todo esto lo significativo para nosotros es que uno de los caminos trazados en esa dirección es un uso crítico de la información que aportan las sociedades vivas a través de la Etnoarqueología.

A partir de las líneas esbozadas durante el desarrollo y crisis de la "Nueva Arqueología", las publicaciones presentadas bajo el epígrafe de "Etnoarqueología" han sido muy abundantes.

En realidad, pueden hacerse infinidad de cosas con datos etnográficos en relación con información arqueológica que estan muy lejos de poderse denominar estudios etnoarqueológicos. De hecho esta vasta bibliografía abarca una amplia gama de premisas teóricas, procedimientos y objetivos.

Los diversos intentos que han sido hechos para poner un poco de orden en todo este panorama van desde esfuerzos mas o menos "taxonómicos" (Stiles, 1977), hasta visiones ambiciosas que pretenden indagar sobre los principios filosóficos subyacentes a los principales trabajos etnoarqueológicos (David, 1992). Su crítica excede por completo el espacio del que disponemos. Bastará aquí decir que de entre todos los trabajos consultados nos llaman la atención los análisis de Susan Kent (1987), y Jorge Onrubia (1987), por cuanto ambos abordan dos cuestiones cruciales para la definición de la naturaleza de la Etnoarqueología, y por consiguiente para el tratamiento de la información etnográfica que hemos recopilado: la analogía y el uniformismo.

Respecto a la primera, ya numerosos autores han llamado la atención manifestando que la analogía nos ha llevado todo lo lejos que podía en el campo de la interpretación, añadiendo que esto ha sido bien poco (Gould, 1980: 36). La propia Susan Kent (1987: 43) se expresa en términos contundentes afirmando que "la analogía etnográfica no es de ningún modo una explicación de nada". Es mas, añadimos nosotros, el procedimiento analógico deja el campo abonado para la multiplicación de las asunciones previas, que lastran y fosilizan nuestro conocimiento.
Onrubia (1987: 61), por su parte, al igual que Kent percibe el carácter crucial que tiene la valoración de la analogía en la resolución de problemas arqueológicos y antropológicos en general, y en concreto en la validación del uso de información etnográfica, afirmando que: "en su uso adecuado radica la legitimación de su interés estratégico para el arqueólogo".

Esta preocupación por el valor de la analogía queda plasmada en ocasiones en la apreciación de una escala en la que poder situar los ejercicios de analogía etnográfica en función de su fiabilidad, para cuya evaluación el principal criterio que se tiene en cuenta es la mayor o menor distancia espacio-temporal de los casos que se pretenden poner en relación.

Pero aunque formulemos tantos criterios restrictivos como queramos para dar como válidas conclusiones derivadas de su uso, la misma base conceptual de la analogía la coloca en un plano de debilidad teórica: continua o discontinua, sistemática o selectiva, una vez se ha perdido la conexión entre las culturas vivas que usamos como referente y las culturas del pasado, ambos tipos de analogía nos obligan a dar el mismo peligroso salto al asumir que las casillas en blanco del registro arqueológico pueden rellenarse con casos "análogos" del presente conocidos de un modo mas completo, procediendo así quizá (por reducción al absurdo) tan alegremente como los ingenieros genéticos de "Parque Jurásico", que reconstruían el ADN de seres extinguidos hace mas de 60 millones de años a partir de la fresca y viva materia prima aportada por anfibios actuales (1).

Desde este punto de vista la necesidad de la analogía dentro de un enfoque determinado define por exclusión lo que es Etnoarqueología. Así la analogía cobraría sentido en iniciativas orientadas a crear reconstrucciones históricoculturales, o simplemente muestrarios de referencias etnográficas para el estudio descriptivo de rasgos concretos del registro arqueológico.

En contraste, un enfoque estrictamente etnoarqueológico, que es lo que nosotros perseguimos, no sólo no tendría por qué, sino que no debería depender de la analogía como núcleo. Para generar un modelo explicativo de mayor alcance es preciso atender a nuestro segundo

(1) Pensamos que el carácter "biológico" de este ejemplo lo hace doblemente significativo para lo que queremos decir. 
concepto clave: el uniformismo, según el cual: "los procesos en el pasado no fueron cualitativamente diferentes a los observados en el presente" (Kent, 1987: 43). Ello implica valorar cuidadosamente los factores ambientales (entendidos en un sentido amplio, no restringidos al marco físico-natural) en los que se definen las formas de explotación económica, el desarrollo de medios tecnológicos o los patrones de distribución espacial. De hecho la Etnoarqueología debe adoptar una perspectiva profundamente ecológica (Gould, 1980: cap. 3), es decir, debe tratar con sistemas complejos en los que numerosas variables interactúan en múltiples direcciones.

No obstante, la validez del procedimiento analógico ha sido defendida también en este plano sistémico (Watson, 1980: 57) (2). Con esto creemos que vamos llegando al fondo de la cuestión.

Una utilización contrastada de la analogía puede orientarnos en el manejo de ciertos conjuntos de rasgos que ponen en relación el presente con el pasado, pero las asociaciones y regularidades que percibimos en los hallazgos arqueológicos no se explican por sí mismas, no hay en ellas implícita una descripción de determinados procesos tecnológicos, intensidad de la explotación del medio o grado de especialización socio-económica. Utilizando la ocurrente expresión de Gould (1980: 45-47), los artefactos (y ecofactos) no son "culpables de asociación" por el mero hecho de aparecer juntos.

En virtud de lo dicho, la analogía no nos ofrece por sí misma resultados concluyentes a un nivel de explicación mas abstracto (Kent, 1987), al cual sólo llegaremos a través de la comparación de los complejos sistemas de adaptación humana, independientemente de su posición espacio-temporal (por ejemplo, mediante la formulación de modelos), de modo que la cuestión no es tanto si nos sirve o no para algo la analogía, como para qué es necesaria y en función de ello qué objetivos, mas o menos amplios, están a nuestro alcance.

(2) Watson (1980: 57): "Las analogías etnográficas podrán ser relativamente complejas y presentadas en un sistema que incluya principios uniformistas bien demostrados o ampliamente aceptados (...) pero los modelos resultantes dependen inevitablemente de analogías derivadas de observaciones de grupos vivos en interacción con su medio ambiente".
Resumiendo, aquello en lo que convergen los principales autores que han abordado el tema es en que el objetivo de la Etnoarqueología es contrastar hipótesis y modelos surgidos en el estudio de regularidades detectadas por la investigación arqueológica, con los datos etnográficos procedentes de sociedades actuales o (como en nuestro caso) del pasado más inmediato, intentando proponer qué adaptaciones humanas del presente, entendidas desde una perspectiva sistémica, son útiles para la comprensión de adaptaciones humanas del pasado.

A este nivel de explicación no hay contradicción entre enfoques tan opuestos como el neoevolucionismo de Binford (1978, 1991, entre otros), el estructuralismo (Kent, 1987) o la Arqueología simbólica y contextual de Hodder (1988, entre otros). Por nuestra parte, queremos expresar nuestra postura dentro de este debate añadiendo que junto a las corrientes teóricas citadas, vemos una escasa presencia de otros planteamientos de base en Etnoarqueología.

Nos referimos al hecho de que las referencias explícitas al tratamiento de la organización social están prácticamente ausentes en la bibliografía etnoarqueológica (Vilá et alii, 1986).

Aún cuando se admitiese en ciertos casos la imposibilidad de alcanzar ese plano social, la comprensión de la interacción hombre-medio nos estaría dando, como mínimo, las condiciones objetivas en las que se desenvolvieron los procesos de producción en el pasado, y por tanto, información sobre la infraestructura económica sobre la que se construyen las relaciones sociales (Ruiz et alii, 1986). La línea quedaría así al menos trazada a la espera de que desarrollos metodológicos ulteriores nos enseñen a superar las limitaciones para llegar a entender y explicar ya no sólo en términos de "pensamiento" (Hodder, 1988 entre otros), flujos energéticos o medios extrasomáticos de adaptación (White, 1949; Alcina Franch, 1989: 44).

Esta perspectiva social e histórica tiene especial importancia en el estudio de sociedades campesinas como la que nos ocupa, cuya organización autosuficiencia y óptimo aprovechamiento del entorno, junto con la respuesta adecuada a condicionantes políticos y sociales que emanan del exterior (rentas, propiedad de la tierra, poder político centralizado...) son dos caras de la misma moneda (Wolf, 1982: 9-31), manifestándose esto con mayor o menor intensi-

T. P., $51, \mathrm{n}^{\circ} 1,1994$ 
dad a través de los diversos cambios culturales que experimenta una determinada región.

\section{EL TRABAJO DE CAMPO: METODOLOGÍA EMPLEADA}

A continuación se expondrán las líneas esenciales del sistema adaptativo tradicional que hemos documentado en el valle del Guadiana Menor. El material se obtuvo fundamentalmente durante dos campañas de trabajo etnográfico en los meses de septiembre de 1992 y 1993. Dichos trabajos consistieron en la realización de entrevistas guiadas mediante cuestionarios temáticos a un total de treinta personas (19 de ellas varones y 11 mujeres), con una media de edad de 70 años. No hemos establecido ningún criterio en la proporción por sexos. Estos cuestionarios trataban por separado los temas de la economía agro-ganadera (tipos y proporción de las especies aprovechadas, fases del ciclo agrícola, estructura de la propiedad...), y la vivienda (selección de materiales, grado de especialización de sus constructores, funcionalidad de las dependencias...).

En las entrevistas hemos marcado como límite cronológico la década de los cincuenta (lo cual evidentemente determina la edad de nuestros informantes), ya que es en este momento cuando se acelera el proceso de transformación de la vida rural en la zona, debido entre otros factores, a la construcción de la primera carretera asfaltada que enlazó las poblaciones de Huesa e Hinojares, y que alteró por completo la antigua dinámica de comunicaciones y desplazamientos.

Además se inició una extensa recopilación de información gráfica sobre arquitectura, sistemas de aprovechamiento del medio, etc. (fotografía, dibujo a escala y cartografía). Paralelamente está en curso una exhaustiva revisión del material documental referente a la evolución histórico-social de nuestra área de trabajo.

En ningún momento pretendemos con la exposición que sigue ofrecer un modelo explicativo definitivo y completo para las adaptaciones de época ibérica en el Guadiana Menor. Simplemente, iniciamos con ella el tratamiento de todo un conjunto de datos muy alentadores en ese camino, un camino que promete ser largo y aún lleno de interrogantes. Además, y con el objeto de mejorar la calidad de la información, en esta primera fase nos hemos centrado en los aspectos de subsistencia y economía como temas monográficos. Sobre todo ello volveremos en el apartado de conclusiones.

\section{SITUACIÓN GEOGRÁFICA}

La zona elegida para realizar el trabajo etnoarqueológico comprende un sector marginal de la llamada "Comarca de la Sierra de Cazorla" (3) que se extiende por la primera y más oriental de las depresiones transversales que cruzan las sierras subbéticas, coincidiendo con el Valle Medio-Bajo del Guadiana Menor, que le sirve de eje principal.

Se trata de una depresión estructural formada por la erosión remontante del mencionado río, origen del típico paisaje de band-lands de la zona, enmarcado por los macizos calcáreos de la Sierra de Cazorla al NE y de Mágina al SE (Higueras, 1961). Administrativamente comprende las localidades de Hinojares, con la aldea de Cuenca; Huesa, con las aldeas de Arroyomolinos, Ceal y Chíllar, además de la aldea de Belerda (Quesada) (Fig. 1).

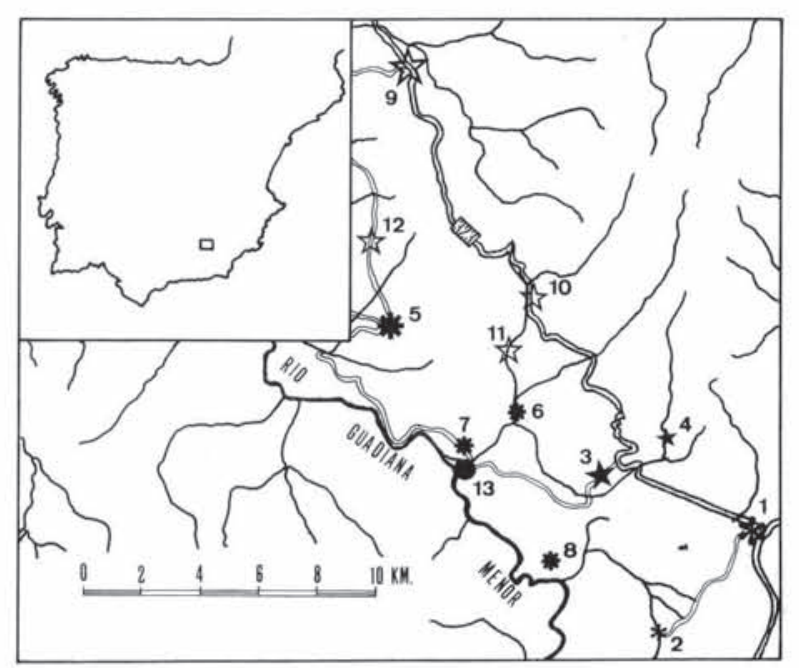

Fig. 1. Situación del área de estudio y ubicación de las poblaciones: 1) Pozo Alcón. 2) Fontanar. 3) Hinojares. 4) Cuenca. 5) Huesa. 6) Arroyomolinos. 7) Ceal. 8) Chíllar. 9) Quesada. 10) Tíscar. 11) Belerda. 12) Los Rosales. 13) Castellones de Ceal.

(3) La comarca de la Sierra de Cazorla está integrada por los términos municipales de Cazorla, Chilluevar, Hinojares, Huesa, la Iruela, Peal de Becerro, Pozo Alcón, Quesada y Santo Tomé. 


\section{EL POBLAMIENTO}

El patrón de asentamiento de esta zona ha sufrido transformaciones a lo largo del tiempo.

Durante la Edad del Bronce se eligen lugares elevados de difícil acceso, fácil defensa, buena visibilidad y amplio control del territorio, cerrando el acceso a las vegas (El Poblezuelo de Chíllar, Cerro Negro y Los Castellones de Ceal y Cuenca) (Sánchez, 1984).

En la Edad del Hierro, aunque no es una zona muy poblada, a juzgar por los datos de que disponemos, se eligen lugares estratégicos próximos a las áreas de explotación y al control de los pasos naturales. El siglo VI a.C. marca el inicio de un asentamiento, los Castellones de Ceal, que va a tener su máximo desarrollo entre los ss. IV-II a.C. Dentro del modelo de los oppida, este yacimiento podría englobarse en la categoría de pequeños poblados (cerca de 1 $\mathrm{Ha}$ ), especializados en el control territorial. Autosuficientes, pero no excedentarios, su relativa riqueza, e incluso a veces su propia existencia no se explicaría sino en función de una estrategía económica dirigida por los pobladores de otras áreas (Chapa, 1992: 318; Ruiz y Molinos, 1993).

No será sin embargo hasta época musulmana cuando se estructure el poblamiento que ha perdurado hasta hoy. Así, Quesada, Huesa, Chíllar, Cuenca y Tíscar aparecen en las fuentes escritas desde el siglo IX al XIII como plazas fuertes que controlan el paisaje (Cruz, 1991: 79; Barceló et alii, 1989), de ahí que se elijan los cerros con mayor intervisibilidad y fácil defensa, situándose los asentamientos en espacios que no resten superficie a los cultivos (Barceló, 1988: 62), como lo demuestran la aparición de restos arqueológicos medievales en la Cortijada de Chíllar, el Cerro de los Castellones de Cuenca, La Peña Negra de Tíscar, etc.

Desde el s. XVI, una vez consolidada la reconquista, las poblaciones se sitúan en laderas, primando en su localización factores de tipo económico (explotación del entorno) y sobre todo, la facilidad de las comunicaciones, sin que intervenga ya el control defensivo. De esta forma aparecen separados de los yacimientos, aunque siempre dentro del mismo territorio, como sucede en Ceal, Hinojares, Cuenca, Arroyomolinos y Huesa.

En la actualidad, la distribución espacial del poblamiento en el Valle Medio-bajo del Guadiana Menor se estructura de manera jerárquica, en función de las divisiones administrativas, mas que del tamaño de los asentamientos. Así tenemos villas (Quesada, Huesa Hinojares y Pozo Alcón), aldeas (Los Rosales, Cuenca, Arroyomolinos y Ceal), cortijadas (Chíllar, Atalayuela, Atochar, Rincón Alto, Rincón Bajo...; cortijos, majadas y chozos (este último no puede considerarse como vivienda).

Generalmente, los núcleos de población aparecen situados en las laderas junto a cauces fluviales. Esta ubicación permite explicar las preferencias en la elección del lugar de asentamiento (Fig. 2):

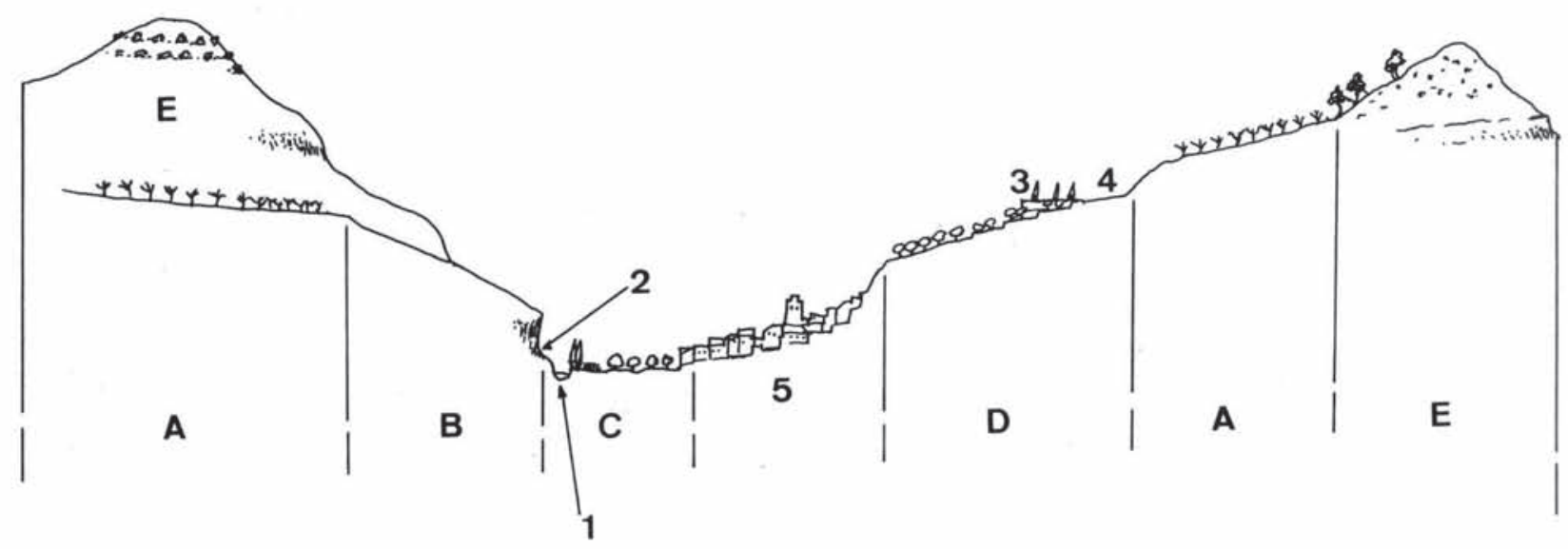

Fig. 2. Esquema del aprovechamiento agrícola del entorno de Hinojares. A) Cultivos de secano en zonas altas amesetadas. B) Improductivo. C) Regadío. D) Bancales para olivar, maiz o frutales. E) Monte bajo. 1) Río Turrilla. 2) Horno.

3) Cementerio. 4) Era. 5) Municipio de Hinojares 
En primer lugar, se busca el control de la vega para su explotación agrícola, escogiéndose zonas algo elevadas, con las viviendas orientadas al río para conseguir, por una parte, el máximo aprovechamiento del valle y por otra, la protección del poblado de los vientos y de posibles riadas. También es importante el control de pasos naturales, en una zona donde lo accidentado del terreno dificulta enormemente las comunicaciones. Muy en relación con esto estaría la necesidad de puntos de descanso y abastecimiento cada cierto intervalo en las vías tradicionales de intercambio y circulación del ganado.

\section{ESTRUCTURA DEL HÁBITAT}

La distribución de los poblados por la ladera de los cerros obliga a una disposición aterrazada de las viviendas, solución natural al problema de la pendiente. El sistema de terrazas se complementa con la construcción de muros de contención, que evitan el deslizamiento de tierras en epoca de lluvias. Esto da lugar a un trazado urbano de calles transversales a la pendiente, adecuándose a las curvas de nivel. Las viviendas se sitúan escalonadas, y a tramos se interrumpen para dejar paso a las empinadas calles que recorren el desnivel. Este tipo de trama urbanística aparece tanto en poblaciones contemporáneas (Arroyomolinos, Hinojares, Cuenca, Ceal) como en poblados de época ibérica, no sólo de nuestra zona (Castellones de Ceal, Jaén), sino de otras áreas: Cerro de la Cruz, Córdoba (Vaquerizo, 1990); San Miguel de Liria, Valencia y Cabezo del Tío Pío, Murcia (Gusi y Olaria, 1984).

La casa es el centro de la vida familiar y la distribución de su espacio está condicionado por una actividad económica de subsistencia. Los modelos son distintos si se trata de una "casa-bloque" o casa "urbana", en la que bajo un mismo techo se reúnen todos los elementos de explotación, o de una "casa disociada" -cortijo y majada- en la que se separa el alojamiento del almacén y la ganadería.

La adaptación de la vivienda a la topografía del terreno ha dado como resultado la aparición de distintas edificaciones dependiendo del constructor. En los "lugares" estudiados hemos podido constatar la existencia de tres tipos de resi- dencia: casa, cueva y casa-cueva (4), aunque en este trabajo únicamente nos ocuparemos de las primeras, puesto que las dos últimas no son características de todas las poblaciones y no se han documentado arqueológicamente.

$\mathrm{La}$ "casa-bloque" tiene generalmente dos plantas; la inferior dedicada a la actividad ganadera (cuadras, chiqueras o pocilgas, gallinero) y la superior, a vivienda y almacén (cocina-hogar y dormitorios- graneros), aunque a veces en la parte inferior puede haber un dormitorio. Además en ocasiones se dispone un tercer piso para almacenaje.

En los cortijos (Lám. IA) y majadas distinguimos en primer lugar la vivienda familiar, que
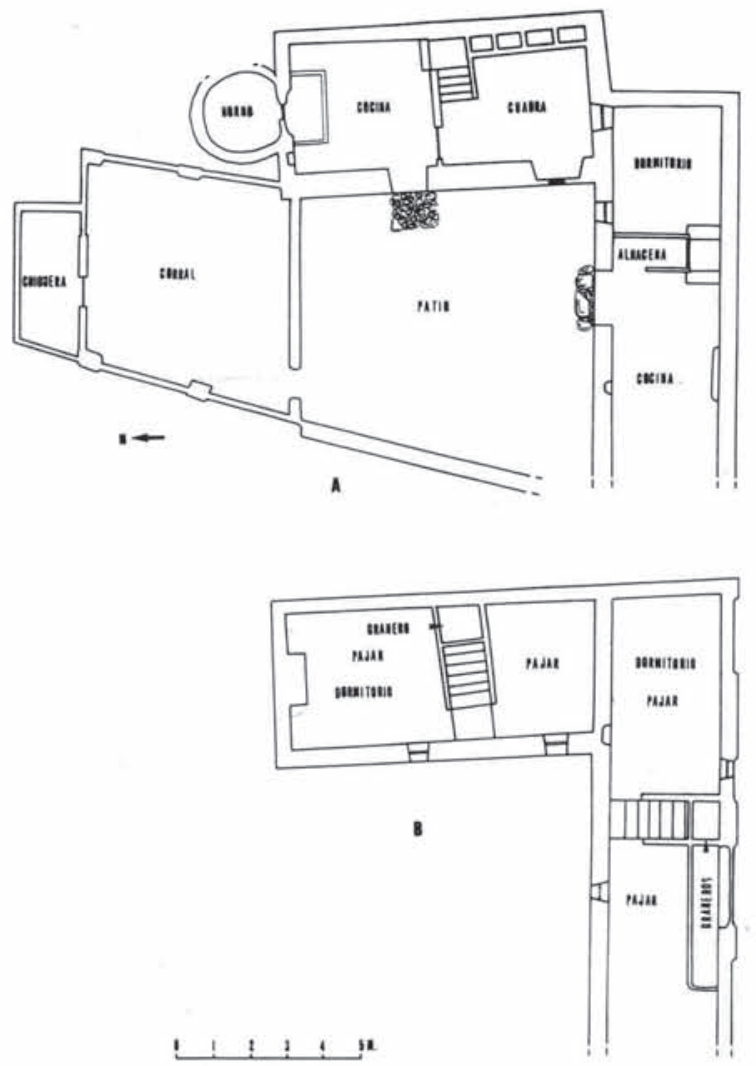

Fig. 3. Cortijo de Ramón González, Ceal.

A) Planta inferior

B) Planta superior

(4) Estas construcciones han sido objeto de un estudio por A. Higueras (1961). Los distintos tipos fijados por este autor no pueden aplicarse per se debido a la enorme variabilidad que ofrece la zona que estudiamos desde los puntos de vista orográfico, climático, agrícola y del sistema de propiedad. 

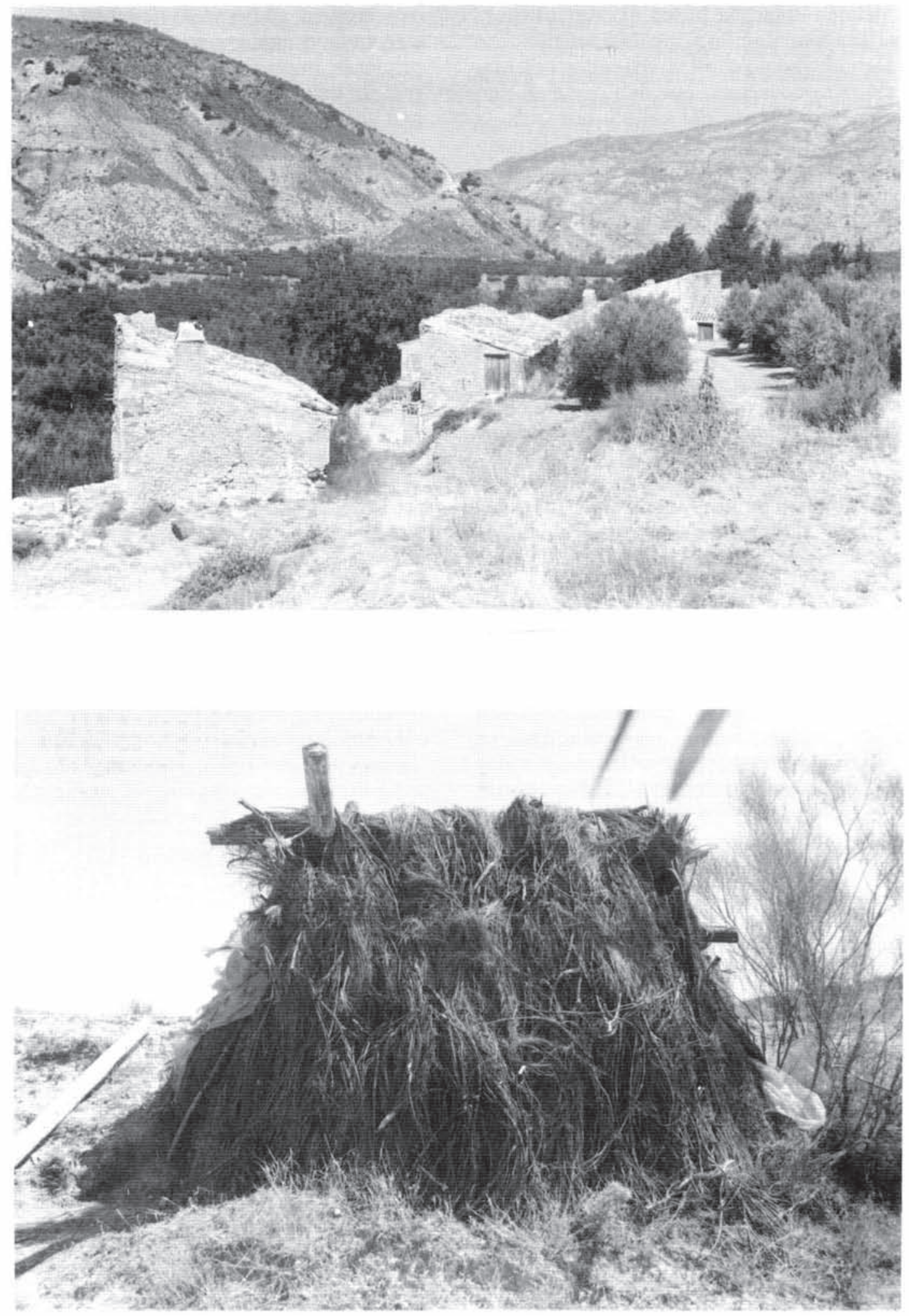

Lám. I. A) Cortijos junto al yacimiento de Castellones de Ceal.

B) Chozo localizado en Hinojares.

T. P., $51, \mathrm{n}^{\circ} 1,1994$ 
suele tener dos plantas (Fig. 3); en la inferior se localiza la cocina con el horno, y el dormitorio principal, y en la parte superior uno o dos dormitorios, utilizados también como graneros. En segundo lugar tenemos la cuadra, a veces con una puerta de acceso directo. Delante o detrás de la casa se sitúa el corral, en torno al cual se disponen las chiqueras, gallineros, leñeras y compartimentos para el ganado.

Del análisis del uso del espacio en las viviendas se deduce que hay estancias con una función muy definida: horno, establo, dormitorio principal, mientras que en otras ocasiones se produce un aprovechamiento para distintas funciones en el mismo espacio. Por ejemplo, ya lo hemos visto, a veces el granero puede utilizarse como dormitorio, y en la cocina-hogar se realizan la mayoría de las actividades domésticas: se cocina, se almacenan víveres, se come, se guarda la vajilla, se recibe a los visitantes, se teje... Además, también en el exterior se llevan a cabo actividades diversas: preparación de las conservas, matanzas, trabajo del esparto, etc. Esta es una cuestión de la que empezamos a tener indicios a partir del estudio microespacial de las viviendas del poblado ibérico, en algunas de cuyas habitaciones se documentan conjuntos cerámicos que hacen referencia a toda una gama de actividades domésticas en el sentido más amplio (transformación de alimentos, consumo, almacenaje...)

Los elementos tradicionalmente utilizados en la construcción los ofrece el entorno inmediato en cantidad y variedad suficiente, con el consiguiente ahorro de tiempo y medios. Estos materiales se someten a un proceso mínimo de transformación, elaborándose sólo aquellos que no pueden emplearse en estado natural, como es el caso del yeso, la cal, el esparto y el barro para hacer adobes y tejas.

En líneas generales, existe una uniformidad en cuanto a técnicas y materiales de construcción en su uso para las diferentes partes de la casa. Los muros exteriores son de mampostería irregular o de cantos de río, aunque en ocasiones se utiliza también el tapial. En la selección de la piedra tiende a preferirse la caliza sobre la cuarcita, y en último lugar el basalto (5), que es

(5) $\mathrm{Al}$ tratarse de una roca que se presenta en grandes bloques con superficies redondeadas resulta demasiado escurridiza y difícil de trabar con argamasa, de ahí que, aún siendo abundante y resistente, se emplee preferentemente en las hiladas inferiores. empleado sobre todo en la cimentación. Los mampuestos van trabados con una argamasa, resultante de mezclar barro, arena, yeso y cal, revocados con este mismo mortero y finalmente encalados.

Los muros interiores siempre están realizados con adobes, siendo asímismo enlucidos de yeso o cal. La cubierta es a una o dos aguas, con tejas al exterior y cañas sobre vigas de madera (pino blanco, sabina o enebro) al interior; la sujeción de las vigas a las cañas y al barro se realiza con ramal de esparto; después se encala o se recubre de una fina capa de yeso para evitar que la humedad o los roedores la dañen. La presencia de yeso también en los suelos evidencia la importancia de dicho elemento en la arquitectura.

En general se practican pocos vanos al exterior, dos o tres ventanas de pequeño tamaño, fundamentalmente destinados a la ventilación. La puerta es de madera rústica de hoja única con dos batientes; ambas se atrancan con cerrojo, el cual se introduce en un agujero practicado directamente en la pared.

Todos estos datos sobre selección de materiales de construcción, distribución del espacio, etc. son pistas que esperamos sean orientativas para empezar a comprender la función y uso de los mismos elementos en el poblado ibérico de Castellones.

En los últimos años se ha producido una gran transformación de la vivienda, debido fundamentalmente al retorno de los emigrantes y a la introducción de nuevas técnicas y recursos. Estas variaciones afectan no tanto al aspecto formal como al funcional: los espacios destinados a los animales (cuadras, pocilgas, gallineros...) se han convertido en dormitorios, cuartos de baño o cobertizos para la maquinaria agrícola.

\section{ECONOMÍA}

La base económica de estas poblaciones es un sistema mixto que combina agricultura, ganadería y recolección. Sólo en algunos casos existe una clara diferencia entre agricultores y ganaderos. Generalmente cada familia dispone de una pequeña huerta, en la que se cultivan hortalizas, legumbres y frutas, alguna parcela de secano, y unos pocos animales: un cerdo, una o 
dos mulas, una cabra, algunas gallinas y, muy excepcionalmente, una vaca.

\section{A) Agricultura y ganadería}

Tomando como referencia la distancia existente entre el lugar de hábitat y la zona de explotación agrícola observamos tres categorías de terrenos, según su rentabilidad y el uso que se hace de ellos. Los campos más próximos, situados entre la población y el cauce del río, son pequeñas extensiones de tierra de regadío dedicadas al cultivo de huerta, donde se plantan gran variedad de productos: lentejas, garbanzos, tomates, pepinos, pimientos, patatas, ajos, cebollas, maíz, frutales y olivar de regadío. El tiempo empleado en llegar a ellas es de escasos minutos. En el límite entre una huerta y otra se construyen chozos con ramas y palos, destinados al almacenamiento provisional de la cosecha durante la recolección (Lám. I-B).

En segundo lugar distinguimos los terrenos de labor dedicados al cultivo de secano, situados en zonas de escasa pendiente y cerros amesetados. En ellos se da el cereal (trigo, cebada, centeno), el olivar de secano y, más recientemente, el almendro. Alcanzar estas parcelas requiere desplazamientos de una media de una a dos horas de duración, que tradicionalmente se han hecho a pie o con caballerías. Cuando las comunicaciones ofrecen una dificultad excesiva, es frecuente que algunas familias habiten de modo permanente en los cortijos, desplazándose hacia los pueblos para lo estrictamente necesario. De hecho, algunos de nuestros informantes han pasado gran parte de su vida en dichos lugares.

En cuanto a la estructura de la propiedad, empezamos a percibir algunos contrastes, aunque no muy acusados, en la extensión de las tierras. Desde luego una diferenciación cualitativa muy neta la establece la posesión de terrenos de regadío, que no está generalizada.

El resto del suelo, que constituye la mayor parte del territorio, es extremadamente abrupto, caracterizado por barrancos, yesares y cerros de greda. Está poblado por vegetación xerófila (esparto, tomillo, romero, mejorana, etc.). En las zonas más alejadas y de mayor altitud crecen pinos (albar, laricio, salgarreño). Estas tierras sin cultivar, de propiedad comunal y hoy apenas explotadas, proporcionaron hasta hace unos años un importante complemento económico, al recolectarse en ellas plantas aro- máticas y medicinales (tomillo, romero, lavanda, mejorana), gramíneas como el esparto, y madera.

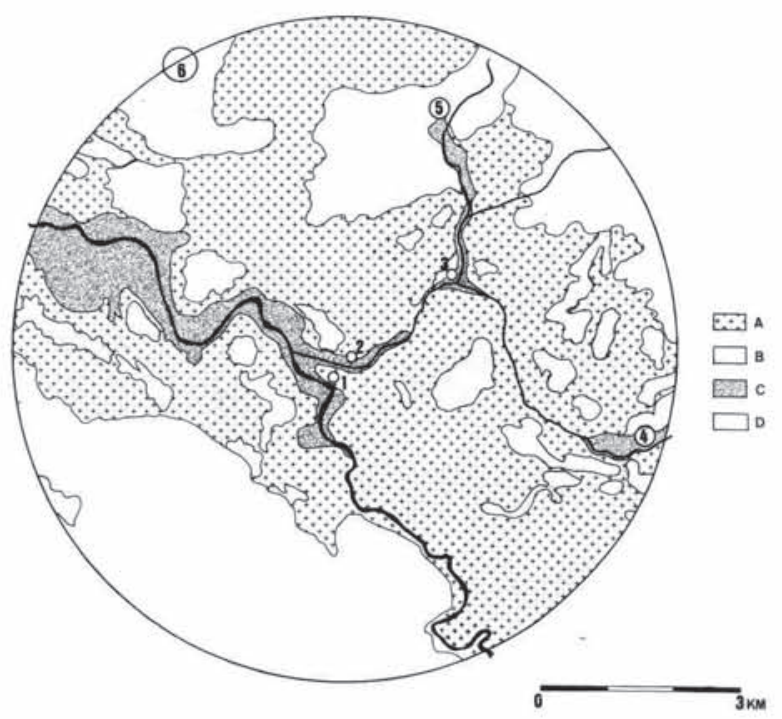

Fig. 4. Esquema de aprovechamientos y cultivos en un radio de $5 \mathrm{Km}$ en torno a Ceal. A) Monte Bajo. B) Secano, C) Regadío. D) Pinos. 1) Castellones de Ceal. 2) Ceal. 3) Arroyomolinos. 4) Hinojares. 5) Belerda. 6) Huesa

El esparto ha sido un elemento económico fundamental pues de su venta se obtenían los medios necesarios para acceder a productos foráneos (calzado y vestido, vajilla doméstica...). La riqueza de esparto de la zona debe de haber hecho posible su explotación y comercialización desde época antigua. Son muy abundantes los restos de esparto trenzado aparecidos en las excavaciones. En los Castellones de Ceal lo encontramos como elemento de sustentación de las ánforas (Chapa et alii, 1987: 353) y como parte integrante de la techumbre de las casas, al igual que hemos visto sucedía en las viviendas actuales. Con estas fibras los habitantes de la zona elaboraban cuerdas, cestas, esparteñas, barjas, esteras, etc. Su explotación ha estado muy controlada hasta hace unos años en que ha dejado de recolectarse de forma sistemática.

Por otra parte, las hierbas aromáticas se han venido empleando para la elaboración de esencias, de aplicación en perfumería, cosmética y medicina. Eran muy importantes las calderas existentes en Hinojares y Chíllar y en los últimos años se están desarrollando de nuevo en 
Huesa. Las esencias resultantes se han dedicado casi exclusivamente a la exportación..

La base de la cabaña ganadera estaba constituida por rebaños de ovejas y cabras; éstos estaban formados por $500-600$ cabezas. Un ejemplo representativo de la estructura de la propiedad del ganado sería el documentado en Arroyomolinos, donde las cabezas de ganado se agrupaban en dos o tres rebaños, conducidos por un pastor; dentro de ellas, algunos propietarios principales poseían una media de 100, 150 cabezas, limitándose la propiedad de los otros a 10, 15 cabezas, que permanecían en el rebaño a cambio de la apropiación de las crías. Quizá en este campo la diferenciación entre propietarios se hace mas neta que en la agricultura.

Dependiendo de la época del año había dos zonas de pasto; en el verano, y una vez recogida la cosecha, el ganado se llevaba a las rastrojeras y en el invierno y la primavera pastaban en la sierra. Estos ganados raramente estaban estabulados; en la sierra pernoctaban al aire libre y en la campiña se encerraban para el sesteo y durante la noche en las majadas, donde se habilitaba una estancia para el pastor. En las épocas de mayor rigor climático (sequías, nevadas) los rebaños transhumaban a otras zonas más propicias.

El principal aporte económico de estos ganados es su venta para aprovechamiento cárnico, bien a través de marchantes que recorrían las aldeas o bien directamente en las vecinas ferias de Baza, Guadix y más excepcionalmente en Ubeda. Ocasionalmente se aprovechaba la carne de los animales que sufrían una muerte violenta. La lana también se vendía, siendo una parte aprovechada para la confección de tejidos.

En resumen, la circulación del ganado ha sido una fuente de beneficios fundamental para poblaciones como Arroyomolinos, ubicadas en un punto intermedio de las rutas ganaderas que procedentes de Toya, conducían a la región andaluza de Baza y Guadix. El camino tradicional iba de Quesada a Huesa, y desde alli pasando por Arroyomolinos e Hinojares alcanzaba Pozo Alcón, a partir de donde la ruta se bifurca, dirigiéndose un ramal hacia Huéscar y otro hacia la depresión de Guadix, lugar de donde provenían la mayoría de los marchantes de ganado. El gran protagonismo de Arroyomolinos como punto intermedio en esta ruta puede comprobarse por las numerosas posadas que tuvo la al- dea (7 en la época de máximo esplendor). Un importante punto de trabajo y discusión en el futuro será el planteamiento de la hipótesis de que el poblado ibérico de los Castellones pudo cumplir una función similar.

Respecto al ganado porcino, hay que reseñar su valor estratégico en la organización de la unidad doméstica campesina

Por último, la caza y la pesca tienen cabida como complemento ocasional de la agricultura y la ganadería. Se han cazado liebres, perdices, conejos, palomas, patos, cabra montesa, jabalí y gamos. Se pescaban truchas, barbos y algunos otros peces, ademas del cangrejo de río. Más frecuente ha sido y aún es la recolección de caracoles, muy abundantes en la zona.

En los análisis de fauna y flora efectuados en el yacimiento de Castellones de Ceal, aparecen representadas las mismas especies de animales y vegetales, tanto domesticadas como silvestres (Chapa, 1984; 1993). Los análisis polínicos y las flotaciones efectuadas han documentado restos de cebada y trigo, (cuya explotación es corroborada además por la abundancia de piedras de molino), lentejas, uvas y olivo, especialmente en las etapas finales del habitat, lo que queda confirmado por la existencia de molinos de aceite (Blanco, 1959: 98). Otras especies vegetales aparecidas son los pinos, que eran empleados como material constructivo en las viviendas y como combustible en los ustrina de la necrópolis (donde aparecen ramas y piñas), fresnos, álamos y esparto. En lo que a fauna se refiere, encontramos una gran variedad de especies, entre las que hay caballos, burros, vacas, ovejas, cabras, cerdos, ciervos, jabalíes, gamos, conejos y gallinas. Algunos de estos animales (gallina y ovicápridos) están relacionados con el ritual funerario en la necrópolis (Chapa et alii, 1991). Desafortunadamente no contamos aún con estudios porcentuales sobre la presencia de cada especímen. No obstante podemos decir en líneas generales, que el ele- mento dominante en la economía de Castellones parece haber sido la ganadería de ovicápridos (Ruiz y Molinos, 1993: cap. 3)

\section{B) Otras actividades}

La producción de sal, yeso y cal han sido tradicionalmente actividades complementarias.

La sal es muy abundante en la zona y resulta indispensable tanto en la dieta humana como animal. Se han documentado dos salinas -Ba- 

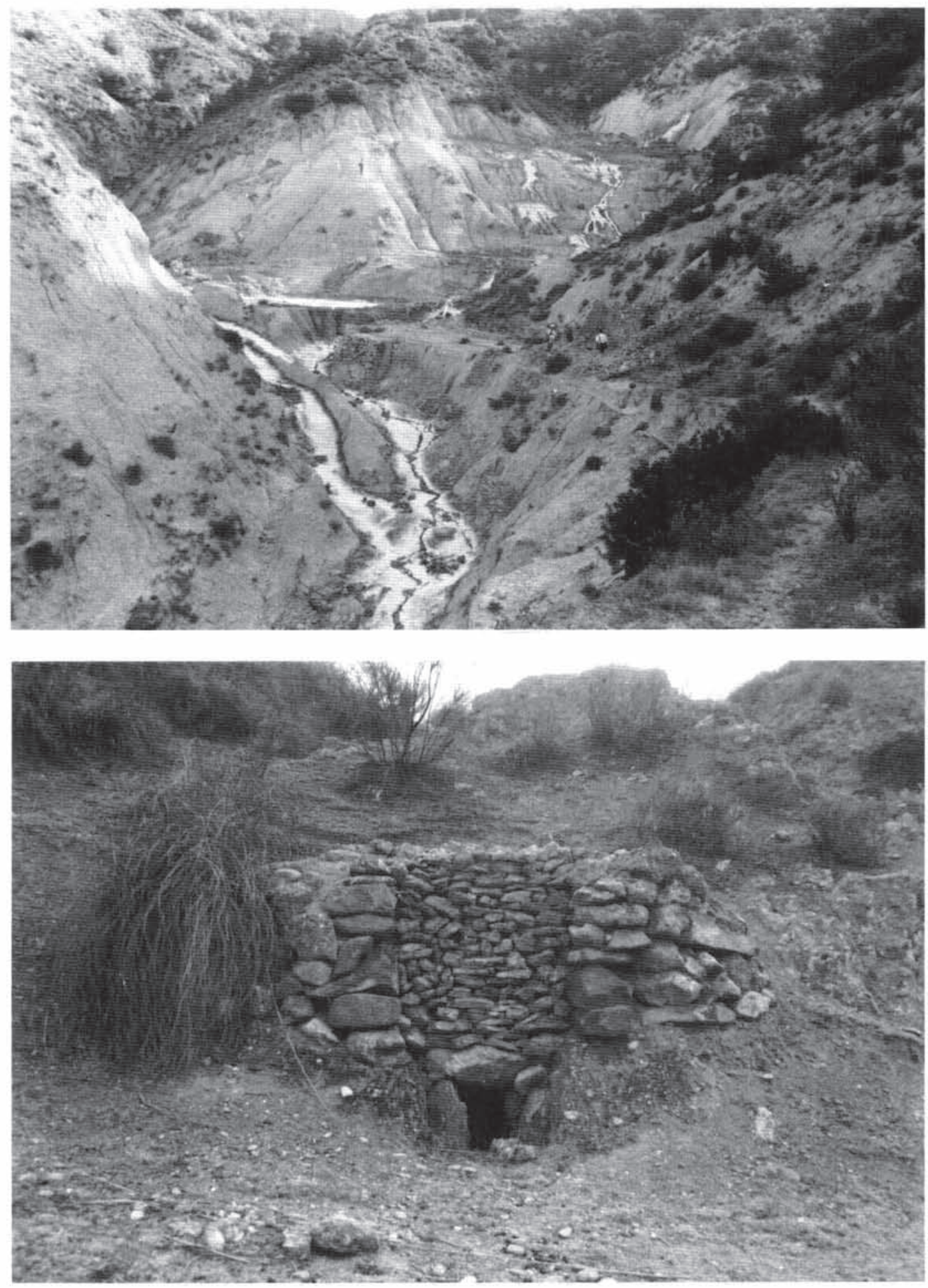

Lám. II. A) Salina del Mesto. Hinojares.

B) Horno para la elaboración de yeso. Chíllar.

T. P., $51, \mathrm{n}^{\circ} 1,1994$ 
rranco de la Salinilla y Salina del Mesto (Lám. IIA) (esta última en explotación al menos desde el s. XVII (6))- de las cuales sólo una se mantiene en funcionamiento. La producción de ambas siempre ha cubierto con creces las necesidades de toda la comarca, por lo que el excedente se exporta hacia las provincias de Granada y Almería. En el pasado, las salinas debieron ser puntos estratégicos controlados por los pobladores de estos territorios (sobre todo si tenemos en cuenta la importancia de la actividad ganadera). Nuestras dos salinas tenían propiedades diferentes. La del Mesto proporciona una sal muy fina que según testimonios de los diferentes informantes era perjudicial para el ganado. Por el contrario, la Salina de Chíllar produce una sal más gorda, buena tanto para personas como para animales.

Como ya hemos visto, el yeso y la cal son materiales asociados a la construcción y se utilizan indistintamente en interiores y exteriores. Para su elaboración existe gran abundancia natural de materia prima. El proceso de fabricación del yeso requiere unas instalaciones de complejidad variable, según el volumen de producción. Se han documentado desde simples agujeros en el suelo, fabricados ex profeso para la construcción de una vivienda concreta (Lam. II-B) hasta yeseras que podríamos calificar como "industriales" dado su rendimiento más elevado y la mayor especialización de los trabajadores.

Las caleras y yeseras se han mantenido en actividad hasta hace pocos años. Las encontramos abandonadas en Chíllar, Huesa y Cuenca. Conocemos arqueológicamente en Castellones de Ceal un uso abundante del yeso para la fabricación de utensilios tales como tapaderas y pequeños recipentes y, ya lo vimos, para la construcción, tanto en la necrópolis como en el poblado.

\section{CONCLUSIONES}

Tal y como señalamos al finalizar la introducción teórica, aún estamos muy lejos de poder proponer resultados concluyentes para nuestro estudio. No obstante, algunas cosas han quedado claras.

(6) Así lo atestigua el hallazgo de una moneda de bronce de Felipe IV.
Desde un punto de vista metodológico queremos insistir en que todas las críticas que hemos realizado respecto al uso de la analogía ni deben interpretarse como un rechazo frontal al empleo de este recurso, ni se contradicen con el ejercicio descriptivo que constituye el núcleo de este artículo. Deberían verse mas bien como una llamada de atención sobre las incongruencias a las que podría llevarnos su mal uso, básicamente una confusión de expectativas y objetivos a la que ya hemos aludido (ver pág. 113). Hemos obtenido, por ejemplo, abundantes indicios respecto a la utilización de los mismos materiales de construcción en viviendas ibéricas y actuales, o sobre la presencia de las mismas especies vegetales y animales en ambos casos, pero entre estas primeras pistas y la reconstrucción de todo un sistema de subsistencia y relaciones sociales dista un abismo, que confiamos se vaya acortando progresivamente. Por lo pronto, pensamos que el giro cualitativo está dado, desde el momento en que pretendemos emplear todo el cuerpo de información que hemos presentado, no como un mero recuento de ejemplos descriptivos que "ilumine" en aspectos concretos nuestro registro arqueológico, sino como plataforma para empezar a comprender la interacción que allí se ha desarrollado con el entorno así como con otras comunidades.

En cuanto a los resultados prácticos, creemos definitivamente confirmado que uno de los ejes básicos en torno al cual ha de girar el planteamiento de hipótesis es la persistencia a lo largo del tiempo en la zona de acentuadas limitaciones ambientales, que confluyen en una estrecha interacción (áreas de cultivo muy reducidas, protagonismo del elemento ganadero, máxima autosuficencia). No obstante -ya lo dijimos- el plano social no debe ser abandonado, y habrá que insistir más en las diferencias internas observadas en la propiedad de tierras y ganado, además de factores externos a estas comunidades campesinas (dependencias en la explotación de la tierra, vinculación a redes de intercambio...). En este último sentido será de enorme interés abordar el problema de la circulación de productos foráneos documentada en el yacimiento de Castellones teniendo presente el tipo de transportes y comunicaciones que ha dominado tradicionalmente en la zona, el grado de variedad de los productos intercambiados, distancias de los contactos... 
Otro camino muy prometedor es el del análisis microespacial, en relación con la polifuncionalidad del espacio documentada etnográficamente. La heterogeneidad y abundancia del contenido artefactual de muchas viviendas ibéricas ya ha sido valorado como posible indicador de la presencia de unidades familiares campesinas (Vaquerizo et alii, 1991: 182). La observación de la distribución de los materiales en el poblado de Castellones empieza á aportar datos alentadores en esta dirección .

Lo que queda desde luego manifiesto a la luz de los diversos factores restrictivos analizados es que la escala de la actividad económica en el curso medio del Guadiana Menor presenta parámetros muy similares en la Segunda Edad del Hierro y en el mundo rural tradicional de, al menos, los últimos cien años. Esta demarcación de la magnitud y naturaleza de los procesos que esperamos entender es de enorme importancia en el análisis arqueológico, y abre un interesante panorama hacia el futuro en la medida en que nos empieza a liberar de los indudables riesgos de distorsión que puede provocar (a menudo inconscientemente) la proyección de conceptos y categorías de nuestro entorno urbano e industrializado sobre el pasado, o mejor dicho y empezando a cambiar de terminología, sobre ese otro mundo, al que nosotros no pertenecemos.

\section{Agradecimientos}

Queremos expresar nuestro agradecimiento a los Drs. Dñ ${ }^{a}$ Teresa Chapa y D. Juan Pereira, por el apoyo prestado durante los trabajos de campo y la elaboración del texto, y a D. Antonio Uriarte, por su desinteresada colaboración. También queremos reseñar la ayuda recibida de D. Marcelino Sánchez y Dña Cruz Sánchez. Ya que sería demasiado prolijo citar aquí a todos y cada uno de nuestros informantes, sirva como prueba de reconocimiento a todos ellos el agradecimiento por su hospitalidad y cooperación a los vecindarios de Hinojares, Ceal, Chíllar, Cuenca y Huesa.

\section{BIBLIOGRAFÍA}

Alcina Franch, J. (1989): "Arqueología antropológica". Akal. Madrid.

T. P., $51, \mathrm{n}^{\circ} 1,1994$
BARCeló, M.; Kirchner, H.; Martí, R. y TORREs, M. (1990): "Sistemas de regadío y asentamientos islámicos en la vertiente sur de la Sierra de Cazorla". III Jornadas de Arqueología Andaluza. Cádiz. Ejemplar mecanografiado.

BINFORD, L. (1978): "Dimensional analysis of prehistoric and site structure: Learning from an Skimo hunting stand". American Antiquity, 43 (3): 330-361

- (1991): "En busca del pasado". (2 ed.). Ed. Crítica. Barcelona.

Blanco, A., (1959): "Excavaciones Arqueológicas en la provincia de Jaén". Boletín del Instituto de Estudios Giennenses. Año VI, 22: 84-126. Jaén.

BONNICHSEN, R. (1973): "Millie's camp: an experiment in archaeology”. World Archaeology, 4 (3): 277-289.

Chang, C. (1983): "Review from Kramer "Village ethnoarchaeology”. American Anthropologist, 85 (4): 950951.

CHAPA, T. (1992): "Caracterización económica de la Alta Andalucía durante la etapa ibérica plena y tardía". En A. Moure Romanillo (ed.): "Elefantes, ciervos y ovicaprinos. Economía y aprovechamiento del medio en la Prehistoria de España y Portugal". Univ. de Cantabria: 315-326.

Chapa, T.; Fernández, M.; Pereira, J. y Ruíz, A. (1984): "Análisis económico y territorial de los Castellones de Ceal (Jaén)". Arqueología Espacial. Coloquio sobre distribución y relaciones entre asentamientos, IV: 223-240. Teruel.

Chapa, T.; Ruíz, A. y Pereira, J. (1987): "Excavaciones en el yacimiento ibérico de Castellones de Ceal (Hinojares, Jaén). Campaña de 1985". Anuario Arqueológico de Andalucía, II, 1985: 353-356. Sevilla.

Chapa, T.; Pereira, J. y Madrigal, A. (e.p.): "El poblamiento de época ibérica en el área del Guadiana Menor: el caso de los Castellones de Ceal (Hinojares, Jaén)". II Jornadas Históricas del Alto Guadalquivir.

DAVID, N. (1971): "The Fulani compound and the archaeologist". World Archaeology, 3 (2): 111-131.

- (1992): "Integrating ethnoarchaeology: a subtle realis perspective". Journal of Anthropological Archaeology, 11: $330-359$

Gould, R. A. (1980): "Living Archaeology". Cambridge University Press. Cambridge.

Gusi, F. y Olaria, C. (1984): "Arquitectura del mundo ibérico". Consejo de Aparejadores y Arquitectos Técnicos de la Comunidad Autónoma Valenciana. Castellón.

Higueras Arnal, A. (1961): "El Alto Guadalquivir. Estudio Geográfico". C.S.I.C. Instituto de Estudios Giennenses. Zaragoza.

Hodder, I. (1988): “Interpretación en Arqueología. Corrientes actuales". Ed. Crítica. Barcelona.

KENT, S. (ed.) (1987): "Method of theory for activity area research: an ethnoarchaeological approach". Columbia Univ. Press.

KRAMER, C. (1982): "Village ethnoarchaeology. Rural Iran in archaeological perspective". Academic Press. New York.

Molina, F.; Aguayo, P.; Fresneda, E. y Contreras, F. (1986): "Nuevas investigaciones en yacimientos de la 
Edad del Bronce en Granada". Homenaje a D. Luis Siret (Cuevas de Almanzora, Almería, 1984): 353-360.

ONRUBIA, J. (1988): "Prehistoria y Etnoarqueología: elementos para una reflexión epistemológica". En J. Onrubia Pintado y E. Manzano Moreno (coord.): "Métodos y tendencias actuales en la investigación geográfica e histórica". Madrid: 57-73.

Ruíz, A; Molinos, M.; Nocete, F. y Castro, M. (1986): "El concepto de producto en Arqueología". Arqueología Espacial. Coloquio sobre el Microespacio, 7: 63-81. Teruel.

Ruíz, A. y Molinos, M. (1993): "Los Iberos. Análisis arqueológico de un proceso histórico". Ed. Crítica. Barcelona.

SÁNCHEZ Ruíz, M. (1984): Estudio arqueológico de los yacimientos del valle del Guadiana Menor, la zona de confluencia con el Guadalquivir desde el Neolítico al Bronce Final. Memoria de Licenciatura Inédita. Univ. de Granada.

SCHIFFER, M. B. (1976): "Behavioral Archaeology". Academic Press. Nueva York.

STILES, D. (1977): "Ethnoarchaeology: a discussion of methods and applications". Man, 12: 87-103.
Trigger, B. G. (1992): “Historia del pensamiento arqueológico”. Ed. Crítica. Barcelona.

VAQUERIZO GIL, D. (1990): "El yacimiento ibérico del Cerro de la Cruz (Almedinilla, Córdoba)”. Imprenta Provincial. Córdoba.

Vaquerizo Gil, D.; Quesada Sanz, F. y Murillo ReDONDO, M. (1991): "Avance al estudio de los materiales arqueológicos recuperados en el yacimiento ibérico de "Cerro de la Cruz". Almedinilla, Córdoba". Anales de Arqueología Cordobesa, 2: 171-224.

Villa Mitja, A.; Argeles Tolo, T. e Yll Aguirre, E. I. (1986): "El microespacio desde una perspectiva etnoarqueológica". Arqueología Espacial 7. Coloquio sobre el Microespacio, 1: 43-49. Teruel.

YeLLEN, J. E. (1977): “Archaeological approaches to the present. Models for reconstructing the past". Academic Press. Nueva York.

WATSON, P. J. (1980): "The theory and practice of ethnoarchaeology with special reference to the Near East". Paleorient, 6: 55-64.

White, L. A. (1949): "The Science of Culture". Farrar Straus. Nueva York.

Wolf, E. R. (1982): “Los campesinos". (3 $3^{\mathrm{a}}$ ed.). Lábor. Barcelona.

T. P., $51, \mathrm{n}^{\circ} 1,1994$ 